Artigo original

\title{
Avaliação da função pulmonar em pacientes com câncer submetidos à quimioterapia
}

Evaluation of the lung function in cancer patients undergoing to chemotherapy

Caroline HeemannVione ${ }^{1}$, Litiele Evelin Wagner ${ }^{1}$, Thaís Ermelinda Schulz Benelli ${ }^{1}$, Marciele Silveira Hopp ${ }^{1}$, Dannuey Machado Cardoso ${ }^{1}$, Dulciane Nunes Paiva ${ }^{1,3}$, Isabella Martins de

Albuquerque ${ }^{4}$

${ }^{1}$ Universidade de Santa Cruz do Sul, Santa Cruz do Sul, RS, Brasil.

${ }^{2}$ Universidade Federal do Rio Grande do Sul, Porto Alegre, RS, Brasil.

${ }^{3}$ Programa de Pós-Graduação em Promoção da Saúde. Universidade de Santa Cruz do Sul, Santa

Cruz do Sul, RS, Brasil.

${ }^{4}$ Universidade Federal de Santa Maria, Santa Maria, RS, Brasil.

Submissão: 08/08/2016

Aceite: 30/09/2016

albuisa@gmail.com

\section{RESUMO}

Justificativa e Objetivo: Alguns agentes neoplásicos usados no tratamento do câncer (CA) causam toxicidade pulmonar e outros importantes efeitos adversos, dessa forma, o presente estudo objetivou avaliar a presença de distúrbio ventilatório obstrutivo (DVO), distúrbio ventilatório restritivo (DVR) ou misto em pacientes com diagnóstico de câncer submetidos a tratamento quimioterápico. Métodos: Trata-se de estudo transversal descritivo que avaliou pacientes com diagnóstico de câncer e submetidos à quimioterapia utilizando os fármacos Doxorrubicina, Bleomicina, Vimblastina, Dacarbazina, Ciclofosfamida, Fluoruracila e Vincristina, sendo excluídos aqueles com diagnóstico de CA de pulmão. Realizado teste de função pulmonar através de análise espirométrica (EasyOne ${ }^{\circledR}$, Switzerland) em pacientes que iniciaram tratamento quimioterápico há mais de 30 dias, tendo sido avaliadas a Capacidade Vital Forçada (CVF), o Volume Expiratório Forçado no primeiro segundo da $\mathrm{CVF}\left(\mathrm{VEF}_{1}\right)$, o Fluxo Expiratório Forçado entre $25-75 \%$ da $\mathrm{CVF}\left(\mathrm{FEF}_{25-75 \%}\right)$ e a Relação $\mathrm{VEF}_{1} / \mathrm{CVFantes} \mathrm{da} \mathrm{sessão} \mathrm{quimioterápica.}$ Resultados: Amostra $(n=18)$ composta por portadores de CA com média de idade de 49,28 $\pm 9,90$ anos e $26,49 \pm 5,67 \mathrm{Kg} / \mathrm{m}^{2}$ onde $44,4 \%$ apresentou padrão espirométrico normal, 27,8\%apresentou DVO, 16,7\% apresentou DVR e 11,1\% apresentou distúrbio ventilatório misto. Conclusão: A quimioterapia resultou em distúrbio pulmonar obstrutivo, restritivo e misto nos portadores de leucemia, câncer de mama e Linfoma de Hodgkin não específico avaliados.

DESCRITORES: Câncer. Quimioterapia. Espirometria.

\section{ABSTRACT}

Background and Objective: Some neoplastic agents used in cancer treatment cause pulmonary toxicity and other important adverse effects, therefore, the present study aimed to evaluate the presence of obstructive lung disease (OLD), restrictive lung disease (RLD) or mixed in patients 
with cancer undergoing chemotherapy. Method: This is a cross-sectional and descriptive study that evaluated patients diagnosed with cancer and undergoing chemotherapy using Doxorubicin, Bleomycin, Vinblastine, Dacarbazine, Cyclophosphamide, Fluorouracil and Vincristine, being excluded those diagnosed with lung cancer. Realized pulmonary function test byspirometric analysis (EasyOne®, Switzerland) in patients who started chemotherapy for over 30 days, it was evaluated the Forced Vital Capacity (FVC), forced expiratory volume in one second FVC (FEV $)_{1}$, forced expiratory flow between $25-75 \%$ of $\mathrm{FVC}\left(\mathrm{FEF}_{25-75} \%\right)$ and the $\mathrm{FEV}_{1} / \mathrm{FVC}$ Relation before the chemotherapy session. Results: Sample $(\mathrm{n}=18)$ composed of cancer patients with average age of $49.28 \pm 9.90$ years and $26.49 \pm 5.67 \mathrm{~kg} / \mathrm{m}^{2}$ on which $44.4 \%$ had normal spirometry standard, $27.8 \%$ had OLD, $16.7 \%$ presented RLD and $11.1 \%$ presented mixed respiratory disorder. Conclusion: Thechemotherapy resulted in obstructive pulmonary disease, restrictive and mixedin patients with leukemia, breast cancer and Hodgkin's lymphoma not specific evaluated.

KEY-WORDS: Cancer. Chemotherapy. Spirometry.

\section{INTRODUÇÃO}

O câncer (CA) tem se consolidado como um problema de saúde pública em todo o mundo. Segundo dados do Instituto Nacional de Câncer (INCA) há estimativa do surgimento de 14 milhões de novos casos e de que, cerca de $60 \%$ dos casos ocorrerão em países em desenvolvimento. No Brasil, a estimativa para os anos de 2016 e 2017 alcança cerca de 600 mil casos novos de câncer. ${ }^{1}$

O tratamento quimioterápico para abordagem das células cancerígenas representa um avanço na cura e no controle do câncer proporcionando aumento da sobrevida e da qualidade de vida, entretanto, os agentes citostáticos têm baixo índice terapêutico e podem produzir efeitos não desejados por atuarem com especificidade relativa sobre as células tumorais. ${ }^{2,3}$ Suas ações farmacológicas podem causar eventos adversos variados, sendo os mesmos divididos em não hematológicos, incluindo as toxicidades cardíacas, pulmonares, hepáticas, gastrointestinais, renais, dermatológicas, disfunções reprodutivas, alterações neurológicas, vesicais, alterações metabólicas, reações alérgicas e a fadiga e os hematológicos que abrangem a leucopenia, anemia, trombocitopenia e a neutropenia febril. ${ }^{4}$

A toxicidade pulmonar geralmente é incomum, porém quando ocorre, pode ocasionar fibrose pulmonar, tosse, dispneia, cansaço, astenia súbita ou progressiva, febre e edema pulmonar após várias semanas de uso dos fármacos, ocasionando complicações secundárias como atelectasias, derrame pleural e infecções. ${ }^{5}$ Tais sinais e sintomas costumam surgir durante ou após vários cursos prolongados de quimioterapia citotóxicas, particularmente aquelas contendo bleomicina, ciclofosfamida, bussulfano, carmustina, mitomicina, metotrexate, procarbazina, clorambucil, com ou sem radioterapia adicional. ${ }^{6}$ Entretanto, deve ser ressaltado que há fatores que 
tornam os indivíduos mais predisponentes ao desenvolvimento de distúrbio ventilatório obstrutivo (DVO) ou distúrbio ventilatório restritivo (DVR) como o emprego de doses cumulativas, a escolha da via de administração, irradiação torácica, idade avançada, função renal e tabagismo. ${ }^{6,7}$

A bleomicina é uma droga quimioterapêutica eficaz, porém com utilização limitada por induzir elevada toxicidade pulmonar. ${ }^{8}$ A ciclofosfamida outro fármaco de eficiência quimioterápica, possui efeito imunossupressor permitindo o seu emprego em uma grande variedade de doenças, como vasculites, pneumonias fibrosantes, glomerulonefrites, granulomatose de Wegener, devendo ser ressaltado que a toxicidade pulmonar possui apresentações na forma aguda (até 6 meses) ou subaguda (mais de 6 meses). ${ }^{6}$

A avaliação da função pulmonar através da espirometria em pacientes com diagnóstico de CA submetidos à quimioterapia torna-se importante para detecção de disfunções respiratórias após início do tratamento quimioterápico. Desse modo, o objetivo da pesquisa foi avaliar a função pulmonar de pacientes com diagnóstico de leucemia, câncer de mama e Linfoma de Hodgkin não específico submetidos a tratamento quimioterápico.

\section{MÉTODOS}

Trata-se de estudo transversal que avaliou os volumes e fluxos pulmonares através de análise espirométrica em portadores de CA submetidos à tratamento quimioterápico. Estudo realizado no Centro de Oncologia Integrado do Hospital Ana Nery, no município de Santa Cruz do Sul - RS, tendo os pacientes assinado o Termo de Consentimento Livre e Esclarecido após aprovação no Comitê de Ética em Pesquisa com Seres Humanos da Universidade de Santa Cruz do Sul - UNISC. Foram incluídos pacientes em tratamento quimioterápico por mais de 30 dias, de ambos os sexos, com idade de 50 a 95 anos e clinicamente estáveis. Excluídos aqueles que realizaram radioterapia, mulheres mastectomizadas, fumantes ou ex-fumantes, com diagnóstico de CA de pulmão ou com pneumopatias, aqueles que não completassem o teste espirométrico ou que apresentassem dispneia durante o exame ou que apresentassem contraindicação à realização do teste espirométrico.

\section{Função Pulmonar}


Os volumes pulmonares foram avaliados através da espirometria (Easy One ${ }^{\circ}$, Modelo 2001, Medizintechnik, Switzerland) onde foram consideradas as variáveis Capacidade Vital Forçada (CVF), Fluxo Expiratório Forçado entre 25-75\% da CVF (FEF 25-75\%), Volume Expiratório Forçado no primeiro segundo $\left(\mathrm{VEF}_{1}\right)$ e a Relação $\mathrm{VEF}_{1} / \mathrm{CVF}\left(\% \mathrm{VEF}_{1}\right)$. A Relação $\mathrm{VEF} / \mathrm{CVF}$ é estadiada de acordo com a idade do indivíduo sendo a normalidade classificada para indivíduos acima de 45 anos em valores acima de $75 \%$ predito e para idosos (acima de 60 anos) em valores acima de 65 e 70\%. Quando reduzida, tal relação indica a presença de distúrbio ventilatório obstrutivo e quando aumentada, indica a presença de restrição. ${ }^{9}$ Durante a realização das medidas, os indivíduos permaneceram sentados e após utilização de um clipe nasal foram orientados a inspirar o máximo possível até a Capacidade Pulmonar Total (CPT) e a expirar sem hesitação para obtenção das medidas. Desta forma, foram realizados três testes, obtendo-se a melhor medida das três curvas fluxo-volume, com critérios dentro da aceitabilidade da espirometria, tendo os valores obtidos sido comparados com os descritos por Pereira et al. e expressos em percentual do predito. ${ }^{10,11}$

Os DVO ocorrem quando há redução da $\mathrm{VEF}_{1}$, com diminuição também da relação $\mathrm{VEF}_{1} / \mathrm{CVF}$ e são classificados em leve (80 a 60 \%predito), moderado (59 a $41 \%$ predito) e severo ( $\leq 40 \%$ predito). Enquanto que os DVR são caracterizados quando a CVF é baixa e há aumento da relação $\mathrm{VEF}_{1} / \mathrm{CVF}$, caracterizando diminuição da $\mathrm{CPT}$, o $\mathrm{FEF}_{25-75 \%}$ pode estar elevado, porém isolado, não diagnostica o distúrbio, classificando-se em leve (80 a 60\%predito), moderado (59 a $51 \%$ predito) e severo ( $\leq 50 \%$ predito). As doenças respiratórias mistas se caracterizam pela redução do $\mathrm{VEF}_{1}$ e da $\mathrm{CVF}$, entretanto a diferença percentual entre ambas é menor que na DVO, assim, se caso a diferença entre o $\mathrm{VEF}_{1}$ e a CVF for igual a doze, trata-se de um distúrbio misto. ${ }^{12}$

\section{Medidas antropométricas}

Para avaliação das características antropométricas foi aferida a estatura através de trena de medidas (Wiso Tecnologia Esportiva ${ }^{\circledR}$, Brasil) fixada na parede e ajustada com um aparelho de nível de bolha, atingindo a altura de $2,20 \mathrm{~m}$, sendo a medida realizada com o paciente descalço e com os pés unidos. O peso corporal foi aferido em balança digital portátil (modelo W801, Wiso Tecnologia Esportiva ${ }^{\circledR}$, Brasil), possuindo plataforma em plástico resistente, capacidade para 180 kg e graduação de 100 gr. As medidas antropométricas foram utilizadas para posterior cálculo do índice de massa corporal (IMC). 


\section{Análise dos dados}

Os dados referentes as doenças de base e ao tempo de tratamento quimioterápico foram obtidos a partir do prontuário do paciente. Os dados foram coletados de modo descritivo e apresentados através das distribuições de frequências simples e percentuais das variáveis antropométricas e espirométricas.

\section{RESULTADOS}

Foram avaliados 18 pacientes (sendo 15 do sexo feminino) portadores de leucemia, CA de mama e Linfoma de Hodgkin não específico, que apresentaram média de idade de 49,28 99,90 anos e IMC de $26,49 \pm 5,67 \mathrm{Kg} / \mathrm{m}^{2}$, sendo a média do ciclo quimioterápico realizada pela amostra de $4,17 \pm 1,54$ ciclos. A amostra avaliada foi submetida aos protocolos ABVD (Doxorrubicina, Bleomicina, Vimblastina, Dacarbazina) com duração do ciclo de 15 dias e com os seguintes ciclos de 21 dias de duração: FAC (Ciclofosfamida, Doxorrubicina, Fluoruracila), AC (Doxorrubicina, Ciclofosfamida), CVP (Ciclofosfamida, Vincristina e Prednisona) e CHOP (Ciclofosfamida, Doxorrubicina, Vincristina e Prednisona)(Tabela 1).

Tabela 1 - Características antropométricas e quimioterápicas.

\begin{tabular}{lc}
\hline Variáveis & $\mathrm{n}=18$ \\
\hline Sexo, feminino $\mathrm{n}(\%)$ & $15(83,30)$ \\
Idade (anos) & $49,28 \pm 9,90$ \\
Peso $(\mathrm{Kg})$ & $69,28 \pm 11,12$ \\
Estatura $(\mathrm{cm})$ & $1,62 \pm 0,08$ \\
$\mathrm{IMC}\left(\mathrm{Kg} / \mathrm{m}^{2}\right)$ & $26,49 \pm 5,67$ \\
Dose $\left(\mathrm{mg} / \mathrm{m}^{2}\right)$ & $6,25 \pm 2,53$ \\
Ciclo & $4,17 \pm 1,54$ \\
Protocolo & \\
$\mathrm{ABVD}_{15}$ & 2 \\
$\mathrm{FAC}_{21}$ & 12 \\
$\mathrm{AC}_{21}$ & 2
\end{tabular}




$\begin{array}{ll}\mathrm{CVP}_{21} & 1 \\ \mathrm{CHOP}_{21} & 1\end{array}$

IMC: Índice de Massa Corporal: $\mathrm{ABVD}_{15}$ : Doxorrubicina, Bleomicina, Vimblastina eDacarbazina com duração de 15 dias; $\mathrm{FAC}_{21}$ :Ciclofosfamida, Doxorrubicina e Fluoruracila com duração de 21 dias; $\mathrm{AC}_{21}$ : Doxorrubicina e Ciclofosfamida com duração de 21 dias; $\mathrm{CVP}_{21}$ : Ciclofosfamida, Vincristina e Prednisonacom duração de 15 dias; $\mathrm{CHOP}_{21}$ : Ciclofosfamida, Doxorrubicina, Vincristina e Prednisona com duração de 21 dias. Dados expressos em média \pm desvio padrão.

Na amostra avaliada, 44,4\% apresentou padrão respiratório normal, 27,8\% apresentou DVO (Leve: 22,2\%; Moderado: 5,6\%), 16,7\% apresentou DVR leve e 11,1\% apresentou padrão ventilatório misto (Tabela 2).

Tabela 2. Valores espirométricos da amostra avaliada.

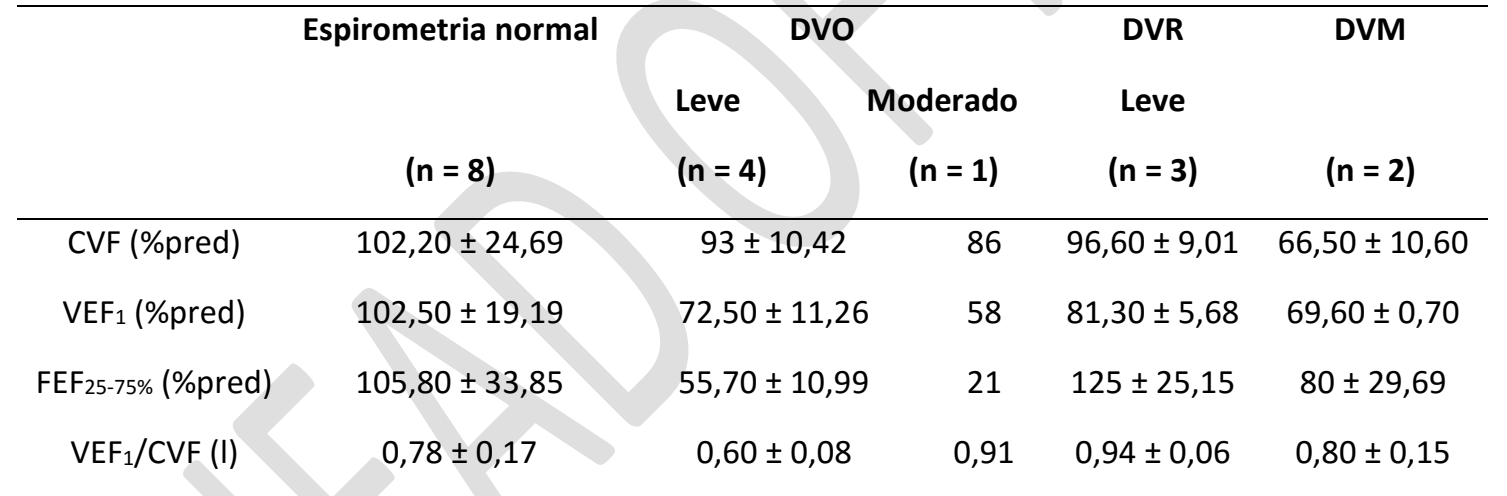

DVO: Distúrbio Ventilatório Obstrutivo; DVR: Distúrbio Ventilatório Restritivo; DVM: Distúrbio Ventilatório Misto; CVF: Capacidade Vital Forçada; $\mathrm{VEF}_{1}$ : Volume Expiratório Forçado no primeiro segundo; $\mathrm{FEF}_{25-75 \%}$ : Fluxo Expiratório Forçado entre $25 \%$ e $75 \%$ da CVF. Dados expressos em média \pm desvio padrão.

\section{DISCUSSÃo}

$\mathrm{O}$ presente estudo demonstrou que pacientes submetidos a quimioterapia evidenciaram alterações na função pulmonar de caráter obstrutivo, restritivo e misto. As lesões pulmonares por quimioterápicos acometem o parênquima pulmonar e as vias aéreas, porém a incidência da toxicidade pulmonar ainda é questionável devido às co-morbidades pulmonares que podem estar presentes, o uso concomitante ou prévio de outros medicamentos, o aspecto nodular gerado como efeito adverso que pode ser confundido com metástases e o fato do comprometimento intersticial apresentar padrão radiológico semelhante à carcinomatose linfática. ${ }^{6,13}$ Geralmente o início de 
sinais e sintomas são semelhantes, entretanto há casos em que podem ocorrer de forma aguda ou vigorosa. Segundo Dimopoulou et al. as manifestações clínicas mais frequentes consistem em dispneia e doença intersticial pulmonar. ${ }^{13}$

As alterações fisiopatológicas advindas da quimioterapia ocorrem a partir do terceiro ciclo quimioterápico, por essse motivo, em nosso estudo, a amostra foi avaliada em média no quarto ciclo. ${ }^{14} \mathrm{Em}$ estudo de revisão de literatura, foi evidenciado que os aspectos clinicos, diagnósticos e terapêuticos das principais pneumopatias induzidas por fármacos, especificamente pela ciclofosfamida, mitomicina e o metotrexate, resultam em um padrão ventilatório restritivo associado à redução da difusão de monóxido de carbono. ${ }^{6}$ Tais evidências corroboram nossos achados, em que 16,7\% pacientes apresentaram padrão ventilatório restritivo.

Outros estudos consideram o uso de pelo menos um dos fármacos hidroxiuréia, daunoblastina, ciclofosfamida, bussulfano, bleomicina emetotrexate como desencadeadores da toxicidade pulmonar. ${ }^{6,15,16}$ A bleomicina resulta em um distúrbio restritivo associado a redução da difusão de monóxido de carbono, CPT, capacidade vital e do VEF1 em cerca de 50\% dos casos. ${ }^{17,18}$ A toxicidade pulmonar pela bleomicina é na maioria das vezes advinda das doses acumulativas, tendo sido evidenciado que doses inferiores a $300 \mathrm{mg}$ raramente causam toxicidade pulmonar e à medida que a dose excede $400 \mathrm{mg}$ há risco de desenvolvimento de toxicidade. ${ }^{19}$

No tratamento de linfoma de Hodgkin, o quimioterápico pode resultar em toxidade pulmonar e redução da capacidade de difusão do monóxido de carbono, sendo esse método eficaz no diagnóstico dos efeitos colaterais do tratamento quimioterápico. ${ }^{20}$ Outro estudo que relata o caso de um paciente com Linfoma não Hodgkin exposto à terapia antineoplásica com ciclofosfamida, doxorrubicina, vincristina, prednisona e rituximabe, ressalta que as lesões pulmonares são frequentes devido aos efeitos colaterais de antineoplásicos, sendo a pneumonia uma das manifestações clinicas presentes neste caso. ${ }^{21}$

Em estudo que avaliou 20 mulheres com diagnóstico de $\mathrm{CA}$ de mama submetidas à quadrantectomia ou a mastectomia foi evidencia da diminuição significativa da CVF $(p=0,04)$ e dopico de fluxo expiratório ( $\mathrm{PFE})(\mathrm{p}=0,02)$ com significativa relação com o tratamento quimioterápico neoadjuvante quando comparado as que apenas realizaram cirurgia de mastectomia ou quadrantectomia. ${ }^{22}$ Há evidências que as modificações pulmonares de mulheres com CA de mama submetidas à quimioterapia com os fármacos farmorubicin, adriamicina, antraciclina, taxano, ciclofosfamida, metotrexato e 5-fluorouracilo resultam em redução significativa da CVF, 
do $\mathrm{VEF}_{1}$ e da capacidade de difusão do monóxido de carbono, enquanto que pacientes tratados apenas com radioterapia local não demonstram redução dos volumes pulmonares pós-tratamento. ${ }^{23}$

Em estudo restrospectivo de análise de prontuários de pacientes com leucemia submetidos aos transplantes de medula óssea (TMO) foi evidenciado que o DVO com CVF reduzida não se encontrava associada à mudanças na evolução clínica dos pacientes avaliados. ${ }^{24}$ Estudo que avaliou a função pulmonar em portadores de mieloma múltiplo evidenciou que a exposição ao bortezomibe resultou na presença do padrão obstrutivo $(\mathrm{p}=0,015)$ enquanto que o padrão restritivo esteve presente na exposição à talidomida $(\mathrm{p}=0,017) .{ }^{26}$ Entretanto, o referido estudo apresentou limitações, pois os pacientes se submeteram a outros agentes quimioterápicos em seu tratamento, sendo que o teste da função pulmonar não foi realizado após cada administração de cada medicação, além de não ter sido avaliada a presença de doenças pulmonares prévias. ${ }^{25}$

A toxicidade pulmonar advinda do tratamento quimioterápico demonstra ser um desafio diagnóstico devido a sua rara ocorrência $(<10 \%)$, podendo se manifestar por uma reação de hipersensibilidade, pneumonite intersticial, edema pulmonar, derrames pleurais, alterações nodulares, bronquiolite obliterante, pneumonia e fibrose pulmonar. ${ }^{26}$ Torna-se importante o esclarecimento dos efeitos desses fármacos sobre a função pulmonar de pacientes oncológicos, uma vez que a saúde e a qualidade vida de tais pacientes devem ser priorizadas. Foi constatado que as drogas quimioterápicas Doxorrubicina, Bleomicina, Vimblastina, Dacarbazina, Ciclofosfamida, Fluoruracila e Vincristinacausaram distúrbios respiratórios obstrutivos, restritivos e mistos em adultos jovens portadores de leucemia, CA de mama e Linfoma de Hodgkin não específico, sendo necessárias mais pesquisas em relação aos efeitos de doses cumulativas das drogas quimioterápicas sobre a função pulmonar.

\section{REFERÊNCIAS}

1- Instituto Nacional de Câncer José Alencar Gomes da Silva (INCA). Incidência de Câncer no Brasil, Estimativa 2016 [Internet]. Ministério da Saúde. Rio de Janeiro: INCA, 2015. [citado 2016 jun 8]. Disponível em: 〈http://www.inca.gov.br/bvscontrolecancer/publicacoes/edicao/Estimativa_2016.pdf>.

2- Guimarães AGC, Anjos ACY. Caracterização sociodemográfica e avaliação da qualidade de vida em mulheres com câncer de mama em tratamento quimioterápico adjuvante. Rev Bras Cancerol 2012;58(4):581-592. 
3- Freitas BN, Neves JB. Efeitos colaterais da quimioterapia: os sentimentos apresentados pelos homens em tratamento. Rev Enferm Integrada 2013;6(1):1064-1073.

4- Gozzo TO, Panobianco MS, Clapis MJ, et al. Toxicidade dermatológica em mulheres com câncer de mama submetidas à quimioterapia. Rev LatAm Enfermagem 2010;18(4): 681687. DOI: https://www.doi.org/10.1590/S0104-11692010000400004.

5- Lacerda MA. Quimioterapia e Anestesia. Rev Bras Anestesiol 2001;51(3):250-270. DOI: https://www.doi.org/10.1590/S0034-70942001000300009.

6- Rubin AS, Perin C, Zibetti L, et al. Pneumopatias induzidas por fármacos. Rev Assoc Med 2000;44(3,4):174-180.

7- Jóna A, Miltényi Z, Póliska S, et al. Effect of Bleomycin Hydrolase Gene Polymorphism on Late Pulmonary Complications of Treatment for Hodgkin Lymphoma. Plos one 2016;11(6). DOI: https://www.doi.org/10.1371/journal.pone.0157651.

8- Sleijfer S. Bleomycin-induced pneumonitis. Chest 2001;120(2):617-624. DOI: https://www.doi.org/10.1378/chest.120.2.617

9- American Thoracic Society. ATS/ERS Task Force: Standardisation of lung function testing. Standardisation of spirometry. Eur Respir J 2005;26:319-338. DOI: https://www.doi.org/10.1183/09031936.05.00034805.

10- Sociedade Brasileira de Pneumologia e Tisiologia (SBPT). II Consenso Brasileiro sobre Espirometria. J Pneumol 2002;28(3):S2-S115.

11- Pereira CAC, Barreto SP, Simões JG, et al. Valores de referência para espirometria em uma amostra da população brasileira adulta. J Pneumol 1992;18(1):10-22.

12- Pellegrino R, Viegi G, Brusasco V, et al. Interpretative strategies for lung function tests. Eur Respir J 2005; 26 (5): 948-68. DOI: https://www.doi.org/10.1183/09031936.05.00035205.

13- Dimopoulou I, Bamias A, Lyberopoulos P, et al. Pulmonary toxicity from novel antineoplastic agents.Ann Oncol 2006;17(3):372-379, 2006.

DOI: https://www.doi.org/10.1093/annonc/mdj057.

14- Abou-Jawde RM, Mekhail T, Adelstein DJ, et al. Impact of induction concurrent chemoradiotherapy on pulmonary function and postoperative acute respiratory complications in esophageal cancer. Chest 2005;128(1):250-5. DOI: https://www.doi.org/10.1378/chest.128.1.250. 
15- Marras TK, Szalai JP, Chan CK, et al. Pulmonary function abnormalities after allogeneic marrow transplantation: a systematic review and assessment of an existing predictive instrument. Bone Marrow Transplant 2002; 30 (9): 599-607. DOI: https://www.doi.org/10.1038/sj.bmt.1703700.

16- Quintas-Cardama A, Perez-Encinas M, Gonzalez S, et al. Hydroxyurea-induced acute interstitial pneumonitis in a patient with essential thrombocythemia. Ann Hematol 1999; 78 (4): $187-8$.

17- Yagoda A, Mukherji B, Young C, et al. Bleomicyn, an antitumoral antibiotic. Ann Intern Med 1972;77:861-870. DOI: https://www.doi.org/10.7326/0003-4819-77-6-861.

18- Froudarakis M, Hatzimichael E, Kyriazopoulou L, et al. Revisiting bleomycin from pathophysiology to safe clinical use. Crit Rev Oncol Hematol 2013;87(1):90-100. DOI: https://www.doi.org/10.1016/j.critrevonc.2012.12.003.

19- Rodriguez JC, Morales MCJ, Casero MAR. Death by bleomycin pulmonary toxicity in ovarian dysgerminoma with pathologic complete response to chemotherapy: A case report. Respir Med Case Rep 2016;18:48-50. DOI: https://www.doi.org/10.1016/j.rmcr.2016.04.0042.

20- Patil N, Paulose RM, Udupa KS, et al. Toxicity of Bleomycin - A Case Series from a Tertiary Care Center in Southern India. J Clin Diagn Res 2016; 10 (4): FR01-FR03. DOI: https://www.doi.org/10.7860/JCDR/2016/18773.7626.

21- Santana AR, Amorim FF, Soares PHA, et al. Insuficiência respiratória aguda causada por pneumonia em organização secundária à terapia antineoplásica para linfoma não Hodgkin. Rev Bras Ter Intensiva 2012;24(4):415-419. DOI: https://www.doi.org/10.1590/S0103507X2012000400020.

22- Abreu ANP, Endres D, Costa AB, et al. Função Pulmonar e Força Muscular Respiratória em Pacientes Submetidas à Cirurgia Oncológica de Mama. Rev Bras Cancerol 2014; 60 (2): 151-157.

23- Spyropoulou D, Leotsinidis M, Tsiamita M, et al. PulmonaryFunctionTesting in Women with Breast Cancer Treated with Radiotherapy and Chemotherapy. In Vivo 2009;23(5):867-71. 
24- Mancuzo EV, Silva WE, Rezende NA. A espirometria na avaliação pré e pós-transplante de medula óssea. J Bras Pneumol 2007;33(1):36-42. DOI: https://www.doi.org/10.1590/S1806-37132007000100009.

25- Bruce JT, Tran JM, Phillips G, et al. Chemotherapeutic Agents Increase the Risk for Pulmonary Function Test Abnormalities in Patients with Multiple Myeloma. Clin Lymphoma Myeloma Leuk 2012;12(5):325-329. DOI: https://www.doi.org/10.1016/j.clml.2012.06.002

26- Livshits Z, Rao RB, Smith SW. Quimioterapia induzida por toxicidade pulmonar. Emerg Med Clin North Am 2014;32(1):167-203. DOI: https://www.doi.org/10.1016/j.emc.2013.09.002. 